\title{
The Effect of Telephone Follow-up on Lifestyle of Patients in Cardiac Care Units
}

\section{Masoumeh Bagheri-Nesami ${ }^{1,2}$, Attieh Nikkhah ${ }^{* 3}$, Sharare Asgari ${ }^{4}$, Soodabeh Aghapoor ${ }^{4}$}

1- Traditional and Complementary Medicine Research Center, Mazandaran University of Medical Sciences, Sari, Iran.

2- World Federation of Acupuncture-Moxibustion Societies (WFAS), Beijing, China.

3- Pediatric Infectious Diseases Research Center, Mazandaran University of Medical Science, Sari, Iran.

4- Hekmat hospital, Social Security Organization, Sari, Iran.

*Correspondence: Mazandaran University of Medical Sciences, Sari, Iran. Tel: +98-9112538813. Email: Atinik1357@gmail.com.

Received July 06, 2019; Accepted December 17, 2019

\section{Abstract}

Background: Telephone follow-up is one of the effective ways to improve lifestyle of cardiac patients. Discharge planning is the development of an individualized discharge plan for the patient prior to leaving hospital, with the aim of improving patient outcomes. This study was done to investigate the effect of telephone follow-up after discharge on the lifestyle of cardiac patients. Methods: This randomized clinical trial was conducted on 154 cardiac patients in cardiac care units in Sari, Iran 2015-2016. Patients were recruited through convenient sampling, then they were allocated randomly in two experimental $(n=77)$ and control $(n=77)$ groups. Before registering, training was provided for both groups of patients. In next step, all the patients were followed up by telephone on the second, fourth and sixth days after the discharge. In addition, the experimental group was followed up by telephone weekly for a month and then monthly for five months. Data was gathered using questionnaire before intervention, as well as one and six months after intervention. Data was analyzed with repeated measures ANOVA, Chi-square and the independent t-test in SPSS- 16.

Results: No statistically significant differences were observed between the two groups in selfcare, physical activity and exercise, diet and stress management score before intervention. However, significant differences were observed between the two groups in self-care $(p<0.001)$, 
physical activity and exercise $(\mathrm{p}<0.001)$, diet $(\mathrm{p}<0.001)$, and stress management $(\mathrm{p}<0.001)$ score after intervention during 6 months.

Conclusions: Telephone follow-up program is effective method promoting the cardiac selfpatients lifestyle. The structured telephone follow-up was important for a holistic approach care in patients.

Keywords: Telephone follow-up, Life style, Heart Diseases, Cardiac Care Units

10.29252/jgbfnm.17.1.67

\section{Introduction}

Cardiovascular diseases are the etiology of 33\% of deaths from non-communicable diseases (1). People with health problems are frequently required to commute between hospital and home during an episode of acute illness (2). Adverse events may occur frequently in the per discharge period, many of which could potentially have been prevented or ameliorated with simple strategies (3). Nurses in the inpatient setting can play a pivotal role in educating patients about the value of outpatient after a cardiac event/procedure(4). Discharge planning is the development of an individualized discharge plan for the patient prior to leaving hospital, with the aim of improving patient outcomes(5). Researches show that hospital discharge program could ddecrease rehospitalization rate in patients 6). Evidence indicated that the period of 4 to 6 weeks after the discharge is a critical time for patients who are at high risk level(7). Elders hospitalized with heart failure, reported more continuity of information about care management and services after discharge planning(8). Discharge services could be reduced hospital utilization within 30 days of discharge(9). According to studies, successful techniques for improving patients' life included self-management interventions(10), life style educational program(11), home- based intervention(12), family based intervention(13) and nurse-led telephone follow up (14). Studies have shown that early outpatient follow-up is associated with lower readmission rates for heart failure $(15,16)$. The results showed that post-discharge nutritional support and telephone follow up in elderly individuals patients could improve functional limitations (17).

Changes in lifestyle after discharge can reduce anxiety, stress and psychological pressures of patients and telephone follow-up is one of the effective ways to improve lifestyle. Patients with cardiovascular disease have other comorbidities for which they may be taking various medications. Patient education reduces the complications of the disease. Carroll and Dorwling in a systematic review study noted that communication, education, patient participation and 
collaboration between medical personnel could improve patient satisfaction and quality of life. The results showed smooth and efficient coordination of this process reduces stress and anxiety for the patient, family, nurse, doctor, hospital and community services(18).

This study was done to investigate the effects of telephone follow-up after discharge on the lifestyle of patients admitted to the cardiac care units.

\section{Methods}

This randomized clinical trial was conducted on cardiac patients of Hekmat hospital in Sari, Iran from 2015 to 2016(Registration No: IRCT201412077494N10). Patients were recruited in the study through convenience sampling considering inclusion criteria. Inclusion criteria include willing to participate in the study, lack of disorientation to place and time, having no history of acute or chronic psychiatric disorder and cancer, and being able to talk and answer the phone. Then, patients was allocated in two experimental $(n=77)$ and control $(n=77)$ group.

The sample size was calculated as 154 according to the mean and standard deviation of diet score in pilot study of 20 patients before and six months after intervention (31.89 \pm 3.34 and $33.94 \pm 4.06$ ) and $95 \%$ the confidence interval, with consideration of the $30 \%$ probability of patient attrition during the study. Using a randomization list, eligible patients were divided into two experimental $(n=77)$ and control $(n=77)$ groups. Randomization list was prepared using Excel RANDBETWEEN function. Written consent was consequently obtained from eligible patients. All participants were informed that participation would be voluntary and they would also be free to decline to answer questions and withdraw from the study at any time.

Before starting the intervention, patients in experimental and control group were taught by a nurse with focusing on safe diet, stress management, drug therapy and daily physical activity. After discharge, patients in both groups were followed up by telephone on the second, fourth and sixth day after discharge. In addition, the experimental group was followed up by telephone for six months; weekly in the first month and each month in next 5 months. The same nurse in the phone call that took 15 minutes explained about the drug (number, frequency, dosage and administration times and side effects of drugs), diet (how to cook and types of diet), activity (amounts and types of activities, problems due to activity) and their habits, such as smoking and addiction. Patients' characteristics including age, gender, diagnosis, marital status, and economic status were assessed using a demographic checklist. Lifestyle was questioned using a 
questionnaire at the beginning of the study, one month and six months later in both groups. Lifestyle questionnaire had 4 dimensions and 49 items including self-care (8 items), exercise and physical activity (3 items), diet (14 items), and stress management (24 items). In a study Cronbach's alpha was used to determine its reliability, which was found to be more than 0.7 in each dimensions of questionnaire (19). Data was analyzed using SPSS Statistics for Windows, version 16 (SPSS Inc., Chicago, Ill., USA). The data were also summarized in Mean, standard deviation and percentage. Normal distribution of variables was assessed using Shapiro-Wilk test.

For normally distributed data repeated measures ANOVA were used. Demographic variables were analyzed using Chi-square and the independent t- test. $\mathrm{P}<0.05$ was considered significant.

\section{Results}

Seventy seven patients were enrolled in each of experimental and control groups. One month after follow up, none of the patients were excluded from the study. But six months later, fifty patients in experimental group were excluded from the study because of death, malignancy and refusing to answer the call. Twenty six patients in control group were also excluded from the study partly due to the same problems (Figure 1).

Thirty six individuals $(46.8 \%)$ in the experimental group and 42 ones $(54.5 \%)$ in the control group were male. The study showed no significant difference in terms of gender between the two groups $(\mathrm{p}=0.425)$. The mean age of patients was $64.06 \pm 12.142$ and $65.79 \pm 12.804$ years in the experimental and control groups, respectively $(\mathrm{p}=0.392)$. Additional demographic and Clinical information are summarized in Table 1.

The Shapiro-Wilk test showed a normal distribution in the experimental and control group for the self-care, physical activity and exercise, diet and stress management, before one month and after six months ( $>$ > 0.05). 


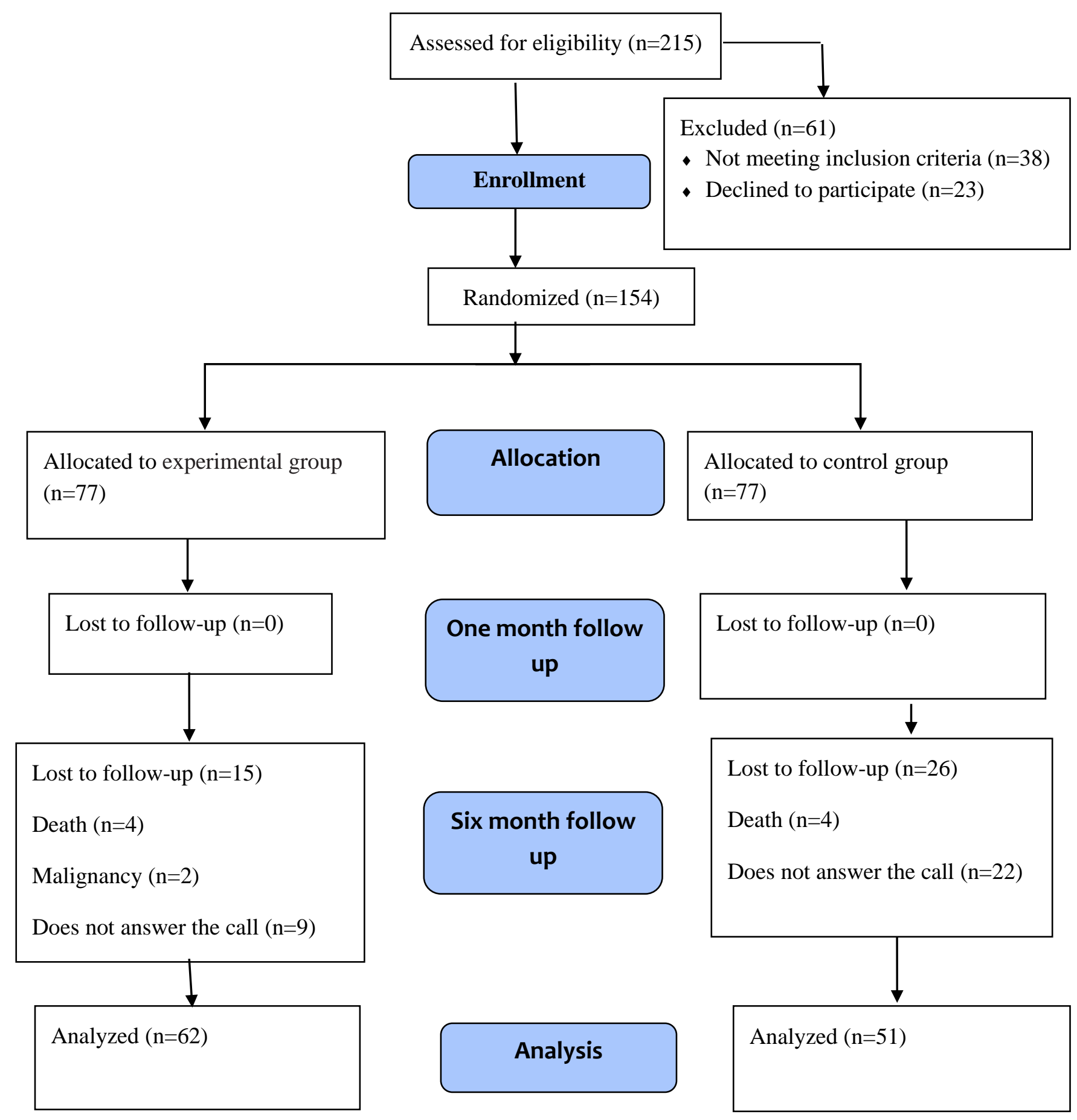

Figure 1. Flowchart of the study's inclusion, allocation and follow-up 
Table 1. Demographic and Clinical characteristics of patients $(n=77)$

\begin{tabular}{|l|c|c|c|}
\hline Gariable & Experimental & Control & p value \\
\hline Age (mean \pm SD) & & & \\
\hline Gender N (\%) & $64.06 \pm 12.142$ & $65.79 \pm 12.804$ & $0.392 *$ \\
Male & $36(46.8)$ & $42(54.5)$ & $0.425 * *$ \\
Female & $41(53.2)$ & $35(45.5)$ & \\
\hline Diagnosis N (\%) & & & \\
Hypertension & $22(28.6)$ & $23(29.9)$ & \\
Acute coronary syndrome & $21(27.3)$ & $19(24.7)$ & \\
Congestive Heart Failure & $5(6.5)$ & $8(10.4)$ & $0.933 * *$ \\
Atrial Fibrillation & $8(10.4)$ & $8(10.4)$ & \\
Myocardial Infarction & $6(7.8)$ & $5(6.5)$ & \\
Other & $14(18.2)$ & $11(14.3)$ & \\
\hline Marital status N (\%) & & $2(2.6 \%)$ & \\
Single & $2(2.6 \%)$ & $64(83.1)$ & $0.436 * *$ \\
Married & $69(89.6 \%)$ & $11(14.3 \%)$ & \\
Widow & $6(7.8 \%)$ & $39(50.6 \%)$ & $0.872 * *$ \\
\hline Economic situation & $38(49.4 \%)$ & $41(53.2 \%)$ & \\
Independent & $39(50.6 \%)$ & & \\
Dependent & & & \\
\hline
\end{tabular}

* T Test

** Chi-squareTest

Repeated measurement test only confirmed significant differences in control groups in terms of physical activity and exercise before starting the follow up, one month and six months after starting the study. Repeated measurement test confirmed significant differences in experimental groups in terms of the physical activity and exercise, diet and stress management, before, one 
month and six months after the intervention. Also, the findings showed that there was no significant difference considering the physical activity and exercise, diet and stress management, before starting and one month after the intervention in experimental and control groups ( $>0.05)$. Figures 2 to 5 show variations of self-care, physical activity and exercise, diet and stress management over time. There were statistically significant differences between two groups in self-care, physical activity and exercise, diet and stress management, before starting, one month and six months after the intervention (Table 2).

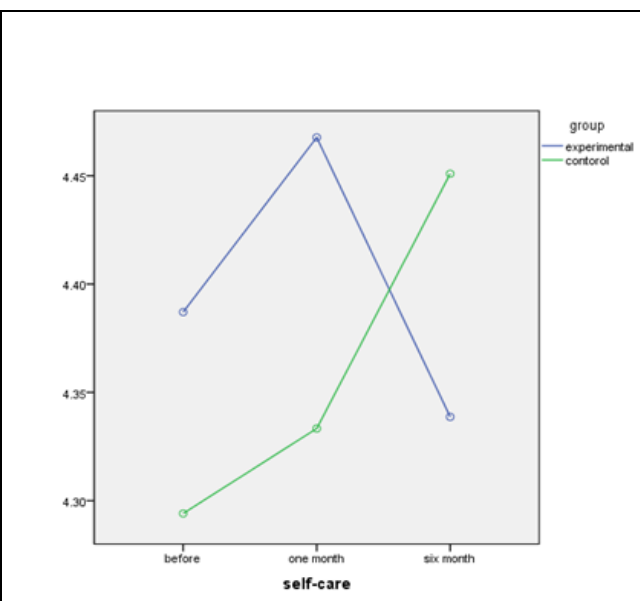

Figure 2. Comparison of the self-care score in three time points in experimental and control groups

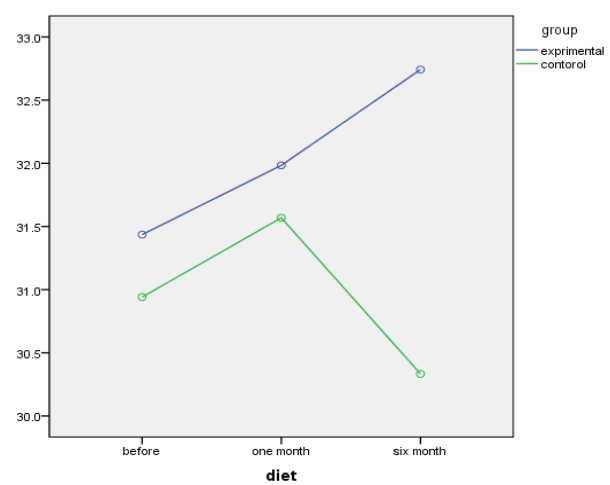

Figure 4. Comparison of the exercise and physical activity score in three time points in experimental and control groups

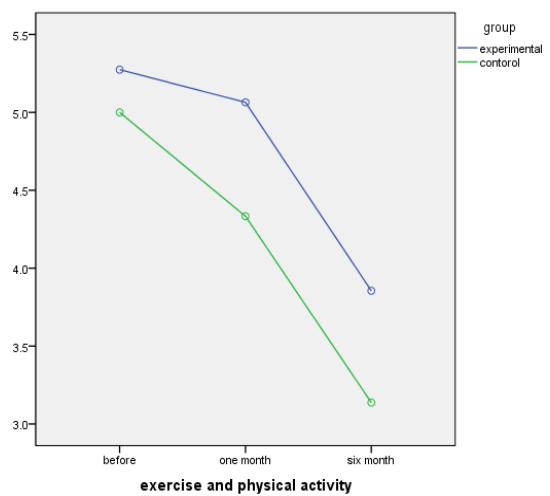

Figure3. Comparison of the self-care score in three time points in experimental and control groups

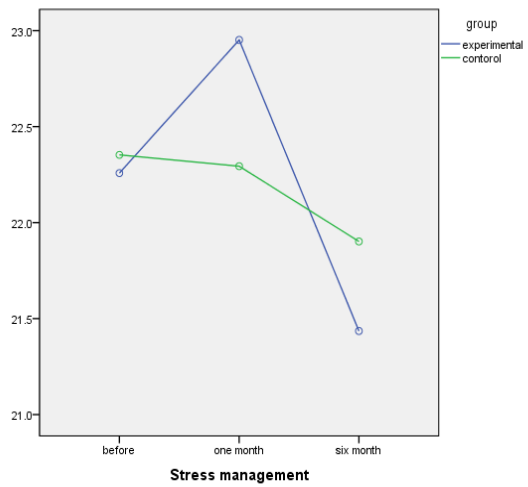

Figure 5. Comparison of the stress management score in three time points in experimental and control groups 
Table 2. Mean score of the lifestyle subscales in three time points in the experimental and control groups

\begin{tabular}{|c|c|c|c|c|c|c|c|}
\hline Group & Exper & mental (Me & $\pm \mathrm{SE})$ & & atrol (Mean= & & \\
\hline Self-care & $4.38 \pm 0.11$ & $4.46 \pm 0.09$ & $4.33 \pm 0.11$ & $4.29 \pm 0.09$ & $4.33 \pm 0.08$ & $4.45 \pm 0.22$ & $\mathrm{P}=0.001$ \\
\hline $\begin{array}{l}\text { Physical activity } \\
\text { and exercise }\end{array}$ & $5.27 \pm 0.38$ & $5.06 \pm 0.37$ & $3.85 \pm 0.33$ & $5.00 \pm 0.47$ & $4.33 \pm 0.36$ & $3.13 \pm 0.35$ & $\mathrm{P}=0.001$ \\
\hline Diet & $31.43 \pm 0.494$ & $31.98 \pm 0.49$ & $32.74 \pm 0.51$ & $30.94 \pm 0.43$ & $31.56 \pm 0.38$ & $30.33 \pm 0.47$ & $\mathrm{P}=0.001$ \\
\hline $\begin{array}{l}\text { Stress } \\
\text { management }\end{array}$ & $22.25 \pm 0.44$ & $22.95 \pm 0.40$ & $21.43 \pm 0.50$ & $22.35 \pm 0.62$ & $22.29 \pm 0.66$ & $21.90 \pm 0.63$ & $\mathrm{P}=0.001$ \\
\hline
\end{tabular}

\section{Discussion}

The results of our study showed that short- term follow-up after discharge could not improve lifestyle of cardiac care unit patients, but long-term follow up could improve all dimensions of cardiac patients' lifestyle in experimental group. Ferrante et. al. assess the effect of long-term telephone intervention on the education and compliance improvement in patients with heart failure. The results of study showed improvement in 1 or more of 3 keys compliance indicators such as diet, weight control, and medication had lower health risks. (20).

Similar to the present study, Shojaee et al (2013) investigated the effect of patient education and telephone follow up for three months on the level of hope in patients suffering from heart failure. Results showed that patient educations at the time of discharge and telephone follow up after the discharge as a cost-effective method to increase hope in patients (21). Wister et. al. in a randomized clinical trial examined the effect of lifestyle intervention in patients with the diagnosis of coronary artery disease. 
Patients in experimental group received a health report card with counselling on smoking, exercise, nutrition and stress from a Tele-health nurse that had certification as clinical exercise specialists. Tele-health counselling occurred within 10 days of the patient receiving the annual report card and every 6 months. The results of this study showed the efficacy of intervention addressing multiple risk factors for primary prevention at 1 year telephone counseling (22).

Aoun et. al. in a study examined the effect of a telephone lifestyle coaching intervention for forty men to evaluate its impact on behavioral change in terms of life quality, body mass index (BMI), physical activity and dietary habits. Results of this study showed significant improvements in lifestyle indicators. Participants were satisfied with the interaction and rated highly the telephone as a medium for coaching. Findings showed that telephone coaching was a feasible means of delivering a lifestyle intervention (23). Also, Courtney et al (2009) reported that exercise strategies and nurse conducted home visit as well as telephone follow-up for 24 weeks could improve quality of life for older adults who is at the risk of hospital readmission (24). In line with our study Hanssen et al (2007) in a study assessed 3 and 6 months telephone follow-up intervention to healthrelated quality of life in patients with acute myocardial infarction after their discharge from hospital. Their results showed that the telephone follow-up improved health-related quality of life and promoted health behavior change in patients after 6 months (25).

Jack et al (2009) documented the effects of telephone follow-up on self-reported preparedness for discharge and frequency of primary care providers' follow-up within 30 days of discharge. Results of this study showed that the participants in the intervention group had a lower rate of hospital utilization than those receiving usual care. Also, the intervention was most effective among the subjects with hospital utilization in the 6 months before index admission(8). Most patients do not meaningfully benefit from early outpatient follow-up. Transitional care resources would be best allocated toward ensuring that the patients with the highest risk receive follow-up within 7 days (25). 
Similar to our short-term results, Altfeld ET. al. Investigated the effects of an enhanced discharge planning intervention for hospitalized older adults. Intervention group received the telephone-based discharge planning intervention that included biopsychosocial assessment and an individualized plan following program protocols. All the patients received a follow-up call during 30 days post-discharge to assess psychosocial needs, patient and caregiver stress, and physician follow-up. The rresults showed that the patients who received the intervention were more likely to communicate and follow up with their physicians. But, the result showed that there were no significant differences between two groups on patients or caregivers stress or hospital readmission(27).

In another study Neelemat ET. al. studied the effect of calcium-vitamin D supplement, energy and protein enriched diet, oral nutritional support with telephone counseling by a dietitian for 3 months post-discharge about physical activities, body weight, functional limitations, physical performance, fat-free mass and handgrip strength. Data were gathered at the hospital admission (baseline) and 3 months after discharge. Results of this study showed that three months of oral nutritional support to malnourished elderly decreased functional limitations and increased body weight (17). It can be questioned if a follow-up of only 3 months was not too short to detect differences on physical performance and physical activities as well. Our research largely confirmed that unlike long-term follow-up, short-term follow-up could not improve lifestyle of patients and thus further randomized trials with longer follow-ups are needed to prove its effectiveness.

\section{Conclusion}

Telephone follow-up program is effective method promoting the cardiac self- patients lifestyle. The structured telephone follow-up were important for a holistic approach care in patients.

\section{Acknowledgements}

The present study was extracted from a research project with the code number of 1697 in 2015. We would like to thank all participants and authorities of research deputy of Mazandaran Uni- 
versity of Medical Sciences for financial support .The authors wish to thank Dr. Khorshidi, Dr. Bahrami and Hekmat hospital nurses who facilitated the sample collection.

\section{References}

1. Mendis S, Puska P, Norrving B. Glob al atlas on cardiovascular disease prevention and control: World Health Organization; 2011.

2. Allen J, Hutchinson AM, Brown R, Livingston PM. Quality care outcomes following transitional care interventions for older people from hospital to home: a systematic review. BMC health services research. 2014.14(1):346. [DOI: 10.1186/1472-6963-14-346]

3. Chaboyer W, Thalib L, Foster M, Ball C, Richards B.Predictors of adverse events in patients after discharge from the intensive care unit. Am J Crit Care. 2008; 17(3):255-263. [DOI:10.4037/ajcc2008.17.3.255]

4. Arena R, Williams M, Forman DE, Cahalin LP, Coke L, Myers J, et al. Increasing Referral and Participation Rates to Outpatient Cardiac Rehabilitation: The Valuable Role of Healthcare Professionals in the Inpatient and Home Health Settings A Science Advisory From the American Heart Association. Circulation 2012; 125(10):1321-9. [DOI:10.1161/CIR.0b013e318246b1e5]

5. Kitzman DW, Brubaker PH, Morgan TM, Stewart KP, Little WC. Exercise training in older patients with heart failure and preserved ejection fraction: a randomized, controlled, single-blind trial. Circ Heart Fail. 2010; 3(6):659-67. [DOI:10.1161/CIRCHEARTFAILURE.110.958785]

6. Sezgin D, Mert H, Özpelit E, Akdeniz B. The effect on patient outcomes of a nursing care and follow-up program for patients with heart failure: A randomized controlled trial. InInt J Nurs Stud. 2017; 70:17-26. [DOI:10.1016/j.ijnurstu.2017.02.013]

7. Naylor MD. Transitional care of older adults. Annu Rev Nurs Res. 2002;20(1):127-47. [DOI: 10.1891/0739-6686.20.1.127]

8. Popejoy LL, Moylan K, Galambos C. A Review of Discharge Planning Research of Older Adults 1990-2008. West J Nurs Res. 2009; 31(7):923-47. [DOI: 10.1177/0193945909334855]

9. Jack BW, Chetty VK, Anthony D, Greenwald JL, Sanchez GM, Johnson AE, et al. A reengineered hospital discharge program to decrease rehospitalization: a randomized trial. Ann Intern Med. 2009; 150(3):178-87. [DOI: 10.7326/0003-4819-150-3-200902030-00007]

10. Romero C, Friedman LC, Kalidas M, Elledge R, Chang J, Liscum KR. Self-forgiveness, spirituality, and psychological adjustment in women with breast cancer. J Behav Med. 2006; 29(1):29-36. [DOI: $10.1007 / \mathrm{s} 10865-005-9038-\mathrm{z}]$ 
11. Schnipper JL, Roumie CL, Cawthon C, Businger A, Dalal AK, Mugalla I, et al. Rationale and design of the Pharmacist Intervention for Low Literacy in Cardiovascular Disease (PILL-CVD) study. Circ Cardiovasc Qual Outcomes. 2010; 3(2):212-9. [DOI:10.1161/CIRCOUTCOMES.109.921833]

12. Giordano A, Scalvini S, Zanelli E, Corrà U, Longobardi GL, Ricci VA, et al. Multicenter randomised trial on home-based telemanagement to prevent hospital readmission of patients with chronic heart failure. Int J Cardiol. 2009; 131(2):192-9. [DOI:10.1016/j.ijcard.2007.10.027]

13. Hu J, Amirehsani KA, Wallace DC, McCoy TP, Silva Z. A family-based, culturally tailored diabetes intervention for Hispanics and their family members. The Diabetes Educator. 2016; 42(3):299-314. [DOI: 10.1177/0145721716636961]

14. Williamson S, Chalmers K, Beaver K. Patient experiences of nurse-led telephone follow-up following treatment for colorectal cancer. Eur J Oncol Nurs. 2015; 19(3):237-43 [DOI:10.1016/j.ejon.2014.11.006]

15. Ryan J, Kang S, Dolacky S, Ingrassia J, Ganeshan R. Change in readmissions and follow-up visits as part of a heart failure readmission quality improvement initiative. Am J Med. 2013; 126(11):989-994. [DOI:10.1016/j.amjmed.2013.06.027]

16. Muus KJ, Knudson A, Klug MG, Gokun J, Sarrazin M, Kaboli P. Effect of post-discharge follow-up care on re-admissions among US veterans with congestive heart failure: a rural-urban comparison. Rur Rem Health. 2010; 10(2):1447.

17. Neelemaat F, Bosmans JE, Thijs A, Seidell JC. Post-discharge nutritional support in malnourished elderly individuals improves functional limitations. J Am Med Dir Assoc. 2011; 12(4):295-301. [DOI:10.1016/j.jamda.2010.12.005]

18. Carroll A, Dowling M. Discharge planning: communication, education and patient participation. Br J Nurs. 2007; 16(14): 882-6. [DOI:10.12968/bjon.2007.16.14.24328]

19. Khalili R, Bagheri-Nesami M, Janbabai G, Nikkhah A. Lifestyle in Iranian Patients with Breast Cancer. J Clin Diagn Re. 2015; 9(7):6-9. [DOI:10.7860/JCDR/2015/13954.6233]

20. Tranmer JE, Parry MJE. Enhancing Postoperative Recovery of Cardiac Surgery Patients a Randomized Clinical Trial of an Advanced Practice Nursing Intervention. West J Nurs Res. 2004; 26(5):515-32. [DOI: 10.1177/0193945904265690]

21. Shojaee A, Tahrir B, Naderi N, Zareian A. Effect of patient education and telephone follow up by the nurse on the level of hope in patients suffering from heart failure. J Nurs Educ. 2013; 2(1):16-26.[Persian]

22. Wister A, Loewen N, Kennedy-Symonds H, McGowan B, McCoy B, Singer J. One-year follow-up of a therapeutic lifestyle intervention targeting cardiovascular disease risk. Can Med Assoc J. 2007; 177(8):859-65. [DOI:10.1503/cmaj.061059] 
23. Aoun S, Osseiran-Moisson R, Shahid S, Howat P, O'Connor M. Telephone lifestyle coaching: is it feasible as a behavioural change intervention for men? J Health Psychol. 2012; 17(2):22736. [DOI: 10.1177/1359105311413480]

24. Courtney M, Edwards H, Chang A, Parker A, Finlayson K, Hamilton K. Fewer Emergency Readmissions and Better Quality of Life for Older Adults at Risk of Hospital Readmission: A Randomized Controlled Trial to Determine the Effectiveness of a 24 Week Exercise and Telephone Follow Up Program. J Am Geriatr Soc. 2009; 57(3):395-402. [DOI:10.1111/j.15325415.2009.02138.x]

25. Hanssen TA, Nordrehaug JE, Eide GE, Hanestad BR. Improving outcomes after myocardial infarction: a randomized controlled trial evaluating effects of a telephone follow-up intervention. Eur J Cardiovasc Prev Rehabil. 2007; 14(3):429-37. [DOI:10.1097/HJR.0b013e32801da123]

26. Jackson CT, Shahsahebi M, Wedlake T, DuBard CA. Timeliness of outpatient follow-up: an evidence-based approach for planning after hospital discharge. Ann Fam Med. 2015; 13(2):115122. [DOI:10.1370/afm.1753]

27. Altfeld SJ, Shier GE, Rooney M, Johnson TJ, Golden RL, Karavolos K, et al. Effects of an enhanced discharge planning intervention for hospitalized older adults: a randomized trial. Gerontologist. 2013; 53(3):430-440. [DOI:10.1093/geront/gns109]

\section{Bibliographic information of this paper for citing:}

Bagheri-Nesami M, Nikkhah A, Asgari SH, et al. The Effect of Telephone Follow-up on Lifestyle of Patients in Cardiac Care Units

J Res Dev Nurs Midw, 2020; 17(1): 81-93.

Copyright (C 2020, Masoumeh Bagheri-Nesami, Attieh Nikkhah, Sharare Asgari, Soodabeh Aghapoor. 